\title{
雷达伺服系统的自适应摩擦力矩补偿控制策略"
}

\author{
姜仁华 ${ }^{1}$ 刘 闯 ${ }^{1}$ 宁银行 ${ }^{2}$ \\ (1. 南京航空航天大学自动化学院 南京 210016; \\ 2. 上海电机学院电气学院 上海 201306)
}

\begin{abstract}
摘要: 外部扰动因素影响下, 时变的摩擦扰动使得雷达伺服系统在低速跟踪扫描或成像定位跟踪时出现爬坡或停止运动等 现象, 严重影响了雷达系统位置跟踪精度。在雷达伺服系统的数学模型的基础上, 通过试验数据分析摩擦力对伺服跟踪精 度的影响。根据机载雷达伺服系统环境多变的特点, 引入温度和扰动影响因子, 改进 LuGre 动态摩擦模型, 设计出自适应 摩擦力矩补偿控制策略, 辨识了摩擦模型的参数, 并结合李雅普诺夫(Lyapunov)稳定理论进行稳定性分析, 得出系统稳定 的控制参数。通过对所提出的控制器进行仿真分析和试验验证, 结果表明新控制器对时变的摩擦力矩具有明显的补偿作用, 可以减少系统低速的爬行现象。在满足雷达系统跟踪精度要求的同时, 可以有效减轻摩擦力矩对雷达伺服系统低速跟踪性 能的影响。
\end{abstract}

关键词: 雷达伺服; 摩擦力矩; 自适应控制; 稳定性分析

中图分类号: TM351

\section{Control Strategy with Adaptive Friction Torque Compensation for Radar Servo System}

\author{
JIANG Renhua $^{1} \quad$ LIU Chuang $^{1} \quad$ NING Yinhang ${ }^{2}$ \\ (1. College of Automation Engineering, Nanjing University of Aeronautics and Astronautics, Nanjing 210016; \\ 2. School of Electric, Shanghai Dianji University, Shanghai 201306)
}

\begin{abstract}
Under the influence of external disturbances, time-varying friction disturbance make radar servo system easily work in the creep behavior or even stop when low-speed tracking, image and locating targets, which seriously affects the system tracking or imaging accuracy. Based on the mathematical model of radar servo system, the influence of friction on the system tracking accuracy is analyzed using the experimental data. In the light of changeable conditions of the servo system, introducing temperature and disturbance influence factors, a control strategy with adaptive friction torque compensation is proposed. For the controller, the LuGre model is improved and its parameters are identified. In addition, according to Lyapunov theory, the control parameters value corresponding to steady conditions are obtained. While meeting the radar servo system accuracy, the proposed controller has obvious compensation for the time-varying friction torque, showed by the simulation and experimental result, which means it can reduce the low-speed creep accident and the influence of friction torque on the radar servo system.
\end{abstract}

Key words: radar servo system; friction torque; adaptive control; steady analysis

\section{0 前言}

在雷达伺服系统进行目标跟踪或成像工作方式 时, 跟踪速率较低, 此时会出现天线扫描不稳定等 现象, 即天线抖动或爬坡等现象, 摩擦力矩是主要 影响因素之一。

* 国家自然科学基金(51377076)、中央高校基本科研业务费专项资金 (XCA17003-06) 和江苏省 “六大人才高峰” (YPC13013) 资助项目。 20181007 收到初稿, 20190320 收到修改稿
在进行快速扫描时, 此时扫描速度较高, 摩擦 力矩与扫描速度成一定的线性关系，对伺服系统的 跟踪性能影响较小; 当进行目标跟踪或成像跟随时, 扫描速度较低, 摩擦力矩表现为较强的非线性, 扫 描出现抖动或爬坡，导致跟踪精度下降或雷达分辨 率下降等现象。此外，由于伺服机构工作环境尤为 严酷, 环境温度变化大、载机的振动/冲击, 导致摩 擦力矩产生较大的波动。为了减少摩擦力矩对伺服 系统的影响, 提高系统的跟踪精度, 研究人员对摩 擦力矩的数学模型和控制方式进行了大量的分析和 
试验研究 ${ }^{[1-9]}$ 。前馈补偿方式是补偿摩擦扰动、提高 系统的跟随精度广泛使用的控制方法之一。文献 [1-2]在摩擦力矩产生机理分析的基础上, 建立摩擦 力矩的动态和静态的数学模型, 并进行了试验研究。 文献[3]采用多采样率前馈摩擦补偿控制器的实现 摩擦补偿, 提高了系统的控制精度。在实际工程应 用环境中, 由于外部环境的变化, 摩擦力矩会出现 动态的变动, 因此可通过摩擦力矩自适应补偿的方 式改善系统的跟踪精度 ${ }^{[4-7]}$ 。文献[4]通过力矩估计值 构建预滑-滑动摩擦力矩模型, 然后采用自适应前 馈补偿方法实现摩擦力矩的补偿, 提高了系统的跟 踪精度。文献[5]在飞行仿真转台动态模型中引入动 态摩擦, 推导出自适应摩擦补偿的鲁棒控制器, 实 现摩擦力矩的自适应补偿。由于摩擦力矩不可测, 文献[8-9]采用了状态观测器实现摩擦力矩的观测, 并通过补偿控制实现系统的跟踪精度的提高。近年 来一些研究学者也提出了自适应反推控制 ${ }^{[10-11]}$ 、模 糊控制 ${ }^{[12-13]}$ 、自抗扰控制 ${ }^{[14]}$ 等控制方法, 实现摩擦 力矩的补偿控制, 并通过试验验证了相关方法的有 效性。

随着研究人员对摩擦模型的深入研究, 20 世纪 90 年代以后, 各种完善的动态摩擦模型的提出, 基 于动态摩擦模型的补偿控制理论和应用成为了热 点, 特别是基于 LuGre 动态摩擦模型的补偿控制研 究 ${ }^{[15-16]}$ 。因此本文展开了基于 LuGre 动态摩擦模型 在雷达伺服系统中的应用研究。由于雷达伺服系统 工作于机载环境下, 工作环境尤为严酷, 伺服系统 的摩擦力矩受温度变化、机械振动/冲击以及载机机 动等多种扰动因素的影响, 而传统的 LuGre 动态摩 擦模型未考虑外部环境因素的影响, 无法实现摩擦 力矩的精确补偿。因此针对外部环境的变化, 研究 人员开展了基于 $L u G r e$ 动态模型的改进研究 ${ }^{[17-19]}$ 。 文献[17]提出了一种反映温度因素的摩擦建模方 法, 实现不同温度下摩擦力矩的精确补偿, 并通过 试验验证了该方法能够满足高精度摩擦力矩的补 偿。文献[18]考虑负载变化引起的 LuGre 摩擦模型 的变化, 根据试验测试数据改进了 LuGre 摩擦模型, 并通过试验测试验证了改进型模型摩擦力矩与真实 摩擦力矩相吻合。

因此，本文在分析了载机环境下摩擦力矩特性 的基础上，借鉴了文献[17-19]的设计思路，引入外 力扰动和温度变化两个影响因子, 改进了 LuGre 动 态摩擦模型, 并结合自适应控制和反推设计法, 设 计了自适应摩擦力矩补偿控制器, 同时采用李雅普 诺夫稳定性理论进行自适应摩擦补偿控制器稳定性 证明和参数设计。最后通过仿真和试验验证本文设
计的控制器有效性和可行性。

\section{1 系统数学模型及摩擦力矩模型}

\section{1 系统数学模型}

本文以某型机载雷达的俯仰轴向为控制对象, 采用表贴式永磁同步力矩电机进行直驱控制。

伺服系统的电机驱动负载主要包括天线负载的 质量不平衡力矩和摩擦力矩等。由于载机在俯仰向 的加速度相对较小, 因此质量不平衡力矩较小, 对 系统的控制精度影响较小，可认为固定负载。

结合永磁同步电机数学模型和 $i_{d}=0$ 矢量控制 方法，可得系统运动方程为

$$
\left\{\begin{array}{l}
J_{L} \ddot{\theta}=k_{T} i_{q}-T_{b}-T_{f} \\
T_{b}=m r a_{J}
\end{array}\right.
$$

式中, $i_{d} 、 i_{q}$ 分别为 $d$ 轴和 $q$ 轴电流; $\theta$ 为伺服位 置反馈信号; $J_{L}$ 为系统转动惯量; $k_{T}$ 为电机转矩常 数; $T_{b} 、 T_{f}$ 分别为不平衡力矩和摩擦力矩; $m$ 为 天线负载质量; $r$ 为天线负载偏心距; $\alpha_{J}$ 为载机加 速度。

\section{2 摩擦力影响分析}

在飞行过程中，雷达伺服系统受到气流波动、 机体振动/冲击以及温度变化等因素的影响, 雷达在 进行低速跟随或目标跟踪时，伺服系统承受的摩擦 力表现为较强的非线性。传统的 PI 控制无法适应动 态非线性负载, 导致跟踪精度变差, 严重时会导致 雷达分辨率下降或目标丢失等现象。图 1 为机载雷 达伺服在地面和空中状态时跟踪误差曲线。

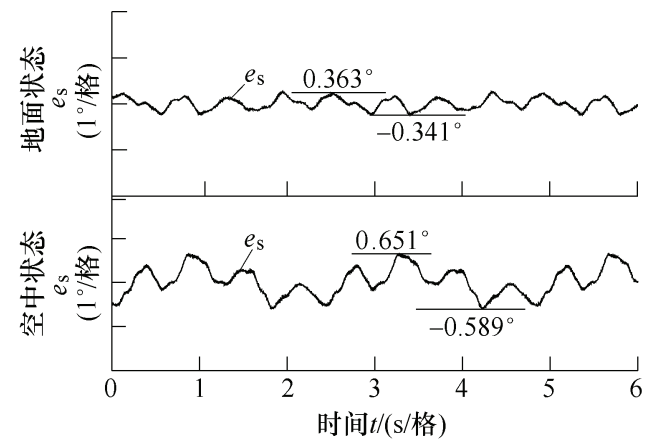

图 1 地面和空中状态跟踪误差对比

在进行目标跟踪状态时, 由于载机飞行相对较 为平稳, 载机 $X Y Z$ 三个轴向的加速度较小, 因此可 忽略负载不平衡力矩的影响。由式(1)可知, 可得在 地面和空中两种状态下，伺服系统承受的力矩主要 为摩擦力矩。

由图 1 可知, 在空中运行状态时由于各种外部 因素的影响，伺服系统低速跟踪时，非线性摩擦力 
矩导致目标跟踪误差增大, 雷达伺服系统跟踪精度 有明显的下降。

\section{2 自适应摩擦力矩补偿控制器设计}

\section{1 改进型动态摩擦力矩模型设计}

近年来, 国内外很多研究人员进行了大量的理 论和试验研究, 提出了许多具有工程实际应用价 值的摩擦力矩模型 ${ }^{[20]}$ 。本文根据机载雷达伺服系 统的应用环境多变的特点, 使用目前工程中广泛 应用的 LuGre 摩擦力矩模型作为系统动态摩擦力矩 模型为

$$
\left\{\begin{array}{l}
T_{f}=\sigma_{0} z+\sigma_{1} \frac{\mathrm{d} z}{\mathrm{~d} t}+\sigma_{2} \dot{\theta} \\
\frac{\mathrm{d} z}{\mathrm{~d} t}=\dot{\theta}-\frac{|\dot{\theta}|}{g_{o}(\dot{\theta})} z \\
T_{z f}=\sigma_{0} z+\sigma_{1} \frac{\mathrm{d} z}{\mathrm{~d} t} \\
T_{v f}=\sigma_{2} \dot{\theta}
\end{array}\right.
$$

式中, $T_{f}$ 为系统摩擦力矩; $T_{z f}$ 为与鬃毛偏移量相关 的摩擦力矩; $T_{v f}$ 为与速度相关的黏滞摩擦力矩; $\dot{\theta}$ 为执行机构的速度; $\sigma_{0} 、 \sigma_{1} 、 \sigma_{2}$ 分别为刚度系数、 阻尼系数和黏滞摩擦因数; $z$ 为䦊毛之间的平均偏 移量, LuGre 模型是通过䦊毛接触对的形成、变形、 接触点的断开及新接触点的形成来表示摩擦行为, 该变量为一个中间变量; $g(\dot{\theta})$ 是斯特里贝克 (Stribeck)效应的参数化表示为

$$
\sigma_{0} g(\dot{\theta})=T_{c}+\left(T_{s}-T_{c}\right) \exp \left(-\left(\dot{\theta} / \dot{\theta}_{s}\right)^{2}\right)
$$

式中, $T_{c}$ 为库仑摩擦力矩; $T_{s}$ 为最大静摩擦力矩; $\dot{\theta}_{s}$ 为斯特里贝克速度。

LuGre 动态摩擦模型只描述了外部环境相对 稳定时的状态, 未反映外部环境变化对摩擦力矩的 影响。

当伺服机构受载机振动或加速度扰动影响时, 模型中的䦊毛接触对受到外部三个轴向的应力影 响, 鬃毛的弹性变形行为发生改变, 从而影响摩擦 行为的大小, 如图 2 所示。

因此选取 $\mu$ 作为载荷力对䦊毛偏移量 $z$ 相关的 摩擦力矩的影响因子, 且 $\mu>0$, 其表达式为

$$
T_{z f}=\mu\left(\sigma_{0} z+\sigma_{1} \frac{\mathrm{d} z}{\mathrm{~d} t}\right)
$$

在空中运行环境下，伺服机构的工作环境温度 存在剧烈的变化, 轴承的润滑剂黏度随温度的变化 而变化, 因此黏度摩擦因数也将随之改变。为此,

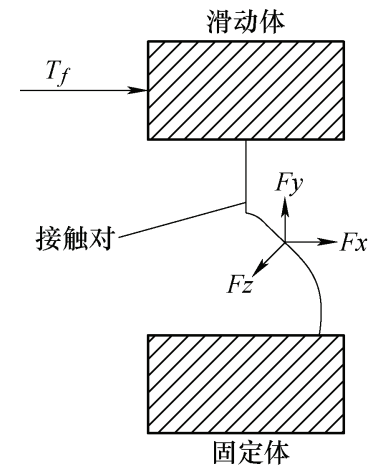

图 2 单鬃毛接触对受力图

本文引入温度影响因子 $\lambda$ 来表示温度变化对黏度摩 擦力矩的变化, 且 $\lambda>0$, 为

$$
T_{v f}=\lambda \sigma_{2} \dot{\theta}
$$

结合式(4)和式(5)可得, 考虑环境因素修正后的 动态摩擦力矩模型为

$$
T_{f}=\mu\left(\sigma_{0} z+\sigma_{1} \frac{\mathrm{d} z}{\mathrm{~d} t}\right)+\lambda \sigma_{2} \dot{\theta}
$$

当雷达伺服系统处于地面静态测试状态时, $\mu=\lambda=1$, 此时的摩擦力矩模型与原 LuGre 模型 一致。

\section{2 自适应摩擦力矩补偿控制器设计}

由式(6)和式(1), 可得改进后的雷达伺服系统机 械运动方程为

$$
\begin{gathered}
J_{L} \ddot{\theta}=k_{T} i_{q}-m r \alpha_{J}- \\
\mu \sigma_{0} z+\mu \sigma_{1} \frac{|\dot{\theta}|}{g(\dot{\theta})} z-\left(\mu \sigma_{1}+\lambda \sigma_{2}\right) \dot{\theta}
\end{gathered}
$$

定义位置跟踪误差 $e_{1}=\theta^{*}-\theta$, 跟踪误差动态 变化为 $\dot{e}_{1}=\dot{\theta}^{*}-\dot{\theta}$, 根据积分反步控制算法 ${ }^{[21]}$, 可 设计速度给定控制信号为

$$
\omega^{*}=k_{1} e_{1}+k_{2} \int_{0}^{T} e_{1}(t) \mathrm{d} t+\dot{\theta}^{*}
$$

式中, 积分项 $\int_{0}^{T} e_{1}(t) \mathrm{d} t$ 用于保证在系统受到外部扰 动时, 位置跟踪接近零; $k_{1} 、 k_{2}$ 为设计参数, 且 $k_{1}>0 、 k_{2}>0$ 。

$\omega^{*}$ 为中间虚拟计算量, 与实际负载速率 $\dot{\theta}$ 之间 存在一定的跟踪误差, 速率跟踪误差方程为

$$
\begin{gathered}
e_{2}=\omega^{*}-\dot{\theta}= \\
\dot{\theta}^{*}-\dot{\theta}+k_{1} e_{1}+k_{2} \int_{0}^{T} e_{1}(t) \mathrm{d} t
\end{gathered}
$$

式中, $\theta^{*}$ 为位置给定指令角。

对式(9)求导并代入式(7), 整理可得

$$
J_{L} \dot{e}_{2}=J_{L} \ddot{\theta}^{*}+J_{L}\left(-k_{1}^{2}+k_{2}\right) e_{1}+
$$




$$
\begin{gathered}
J_{L} k_{1} e_{2}-J_{L} k_{1} k_{2} \int_{0}^{T} e_{1}(t) \mathrm{d} t+ \\
\mu \sigma_{0} z-\mu \sigma_{1} \frac{|\dot{\theta}|}{g(\dot{\theta})} z+\left(\mu \sigma_{1}+\lambda \sigma_{2}\right) \dot{\theta}- \\
k_{T} i_{q}-m r \alpha_{J}
\end{gathered}
$$

由于鬃毛之间的平均偏移量 $z$ 是不可测量, 为 了观测鬃毛的平均偏移量, 设计了两个非线性状态 观测器估计状态量 $z$, 观测器方程为

$$
\left\{\begin{array}{l}
\frac{\mathrm{d} \hat{z}_{0}}{\mathrm{~d} t}=\dot{\theta}-\frac{|\dot{\theta}|}{g(\dot{\theta})} \hat{z}_{0}+\tau_{0} \\
\frac{\mathrm{d} \hat{z}_{1}}{\mathrm{~d} t}=\dot{\theta}-\frac{|\dot{\theta}|}{g(\dot{\theta})} \hat{z}_{1}+\tau_{1}
\end{array}\right.
$$

式中, $\hat{z}_{0} 、 \hat{z}_{1}$ 为鬃毛平均偏移量的估计值; $\tau_{0} 、 \tau_{1}$ 为状态观测器的补偿量。

根据式 (10), 以及李雅普诺夫函数 $V_{1}=\frac{1}{2} e_{1}^{2}+\frac{1}{2} e_{2}^{2}+\frac{1}{2} k_{2}\left(\int_{0}^{T} e_{1}(t) \mathrm{d} t\right)^{2}$, 构造自适应控制 律, 得位置环输出的给定 $q$ 轴电流为

$$
\begin{gathered}
i_{q}=\frac{J_{L}}{k_{T}}\left(1-k_{1}^{2}+k_{2}\right) e_{1}+\frac{J_{L}}{k_{T}}\left(k_{1}+k_{3}\right) e_{2}- \\
\frac{J_{L}}{k_{T}} k_{1} k_{2} \int_{0}^{T} e_{1}(t) \mathrm{d} t+\frac{\sigma_{0}}{k_{T}} \hat{z}_{0} \hat{\mu}- \\
\frac{\sigma_{1}}{k_{T}} \frac{|\dot{\theta}|}{g(\dot{\theta})} \hat{z}_{1} \hat{\mu}+\frac{\sigma_{1}}{k_{T}} \dot{\theta} \hat{\mu}+\frac{\sigma_{2}}{k_{T}} \dot{\theta} \hat{\lambda}+ \\
\frac{J_{L}}{k_{T}} \ddot{\theta}^{*}+\frac{m r \alpha_{J}}{k_{T}}
\end{gathered}
$$

式中, $\tilde{z}_{0} 、 \tilde{z}_{1}$ 为实际值与观测值的误差; $\tilde{\mu} 、 \tilde{\lambda}$ 表 示真实值与估计值之间的误差。

根据李雅普诺夫第二法判断系统的稳定性, 构 造李雅普诺夫闭环函数进行系统稳定性分析, 其表 达式为

$$
V=V_{1}+\frac{1}{2 r_{0}} \tilde{\mu}^{2}+\frac{1}{2 r_{1}} \tilde{\lambda}^{2}+\frac{1}{2} \mu \tilde{z}_{0}^{2}+\frac{1}{2} \mu \tilde{z}_{1}^{2}
$$

式中, $r_{0} 、 r_{1}$ 为自适应增益, 且 $r_{0}>0 、 r_{1}>0$ 。由 于 $k_{2} 、 r_{0} 、 r_{1} 、 \mu 、 \lambda$ 均为正实数, 因此确定 $V$ 是 正定的。

根据式(9)、式(11)和式(12), 计算李雅普诺夫对 时间的微分

$$
\begin{gathered}
\dot{V}=-k_{1} e_{1}^{2}-k_{3} e_{2}^{2}-\mu \frac{|\dot{\theta}|}{g(\dot{\theta})} \tilde{z}_{0}^{2}-\mu \frac{|\dot{\theta}|}{g(\dot{\theta})} \tilde{z}_{1}^{2}+ \\
\tilde{\mu}\left(\frac{\sigma_{1}}{J_{L}} \dot{\theta} e_{2}-\frac{1}{r_{0}} \dot{\hat{\mu}}\right)+\tilde{\lambda}\left(\frac{\sigma_{2}}{J_{L}} \dot{\theta} e_{2}-\frac{1}{r_{1}} \dot{\hat{\lambda}}\right)+
\end{gathered}
$$

$$
\mu \tilde{z}_{0}\left(\frac{\sigma_{0}}{J_{L}} e_{2}-\tau_{0}\right)-\mu \tilde{z}_{1}\left(\frac{\sigma_{1}}{J_{L}} \frac{|\dot{\theta}|}{g(\dot{\theta})} e_{2}-\tau_{1}\right)
$$

选择各未知参数的自适应规律和状态观测器的 误差补偿项如式(15)所示

$$
\left\{\begin{array}{l}
\dot{\hat{\mu}}=\frac{\sigma_{1}}{J_{L}} r_{0} \dot{\theta} e_{2} \\
\dot{\hat{\lambda}}=\frac{\sigma_{2}}{J_{L}} r_{1} \dot{\theta} e_{2} \\
\tau_{0}=\frac{\sigma_{0}}{J_{L}} e_{2} \\
\tau_{1}=\frac{\sigma_{1}}{J_{L}} \frac{|\dot{\theta}|}{g(\dot{\theta})} e_{2}
\end{array}\right.
$$

由式(14)可得李雅普诺夫函数对时间的微分方 程的简化表达式为

$$
\dot{V}=-k_{1} e_{1}^{2}-k_{3} e_{2}^{2}-\mu \frac{|\dot{\theta}|}{g(\dot{\theta})} \tilde{z}_{0}^{2}-\mu \frac{|\dot{\theta}|}{g(\dot{\theta})} \tilde{z}_{1}^{2}
$$

由于 $k_{1} 、 k_{3} 、 \mu$ 为正实数, 且 $g(\dot{\theta})>0 、|\dot{\theta}| \geqslant 0$, 可得 $-\infty<\dot{V} \leqslant 0$, 因此 $V$ 有界且单调不增, 根据李 雅普诺夫定理可知, 采用式(12)和式(15)的自适应摩 擦补偿控制律能够够保证系统是全局渐近稳定的。 式(13)和式(16)中的组成变量 $e_{1} 、 e_{2} 、 \tilde{\mu} 、 \tilde{\lambda} 、 \tilde{z}_{0}$ 、 $\tilde{z}_{0}$ 均为有界变量, 即位置和速度跟踪误差全局有 界。由 Barbalat 引理 ${ }^{[22]}$ 可证明当 $t \rightarrow \infty$ 时, $e_{1} \rightarrow 0$ 、 $e_{2} \rightarrow 0$, 即位置和速度跟踪误差渐近收玫到零。综 上可得基于自适应摩擦补偿控制器框图见图 3。

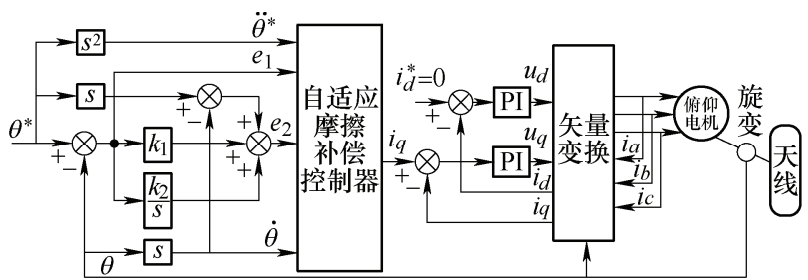

图 3 自适应摩擦补偿控制器系统框图

\section{LuGre 动态摩擦模型参数辨识}

LuGre 动态摩擦力矩模型包含两部分特性, 一部分为稳态摩擦特性, 在稳态或高速运行时的 摩擦特性; 一部分为动态摩擦特性, 主要表征系 统在动静转换临界状态的摩擦特性 ${ }^{[1]}$ 。本文在进 行摩擦特性参数辨识时, 分为稳态参数辨识和动 态参数辨识。

\section{1 稳态摩擦特性参数辨识}

稳态时, $\mathrm{d} z / \mathrm{d} t=0$, 由式(2)和式(3)可得稳态摩 
擦力矩方程为

$$
T_{f}=\left[T_{c}+\left(T_{s}-T_{c}\right) \exp \left[-\left(\dot{\theta} / \dot{\theta}_{s}\right)^{2}\right]\right] \operatorname{sgn}(\dot{\theta})+\sigma_{2} \dot{\theta}
$$

由式(17)可知, 稳态摩擦特性可通过参数 $T_{c}$ 、 $T_{s} 、 \sigma_{2} 、 \dot{\theta}_{s}$ 来表述。本文通过样机试验验证曲线拟 合的方式, 辨识稳态摩擦力矩模型的参数。

在实际系统中, 由于天线负载存在质心偏移的 现象, 导致测试力矩中包含了负载不平衡力矩, 因 此测试中采用等惯量质心平衡负载进行摩擦力矩 测试。

在测试过程中, 通过采样伺服系统依次给定 $\pm 0.5 \sim \pm 10^{\circ} / \mathrm{s}$ 速度信号情况下的输出 $q$ 轴电流, 根 据电机转矩常数得出相应的力矩大小。最后通过最 小二乘拟合方法对俯仰轴正反转运动测量的力矩数 据进行拟合, 得到稳态摩擦特性的四个参数 $T_{c} 、 T_{s}$ 、 $\sigma_{2} 、 \dot{\theta}_{s}$ 的值。试验测试波形如图 4 所示。

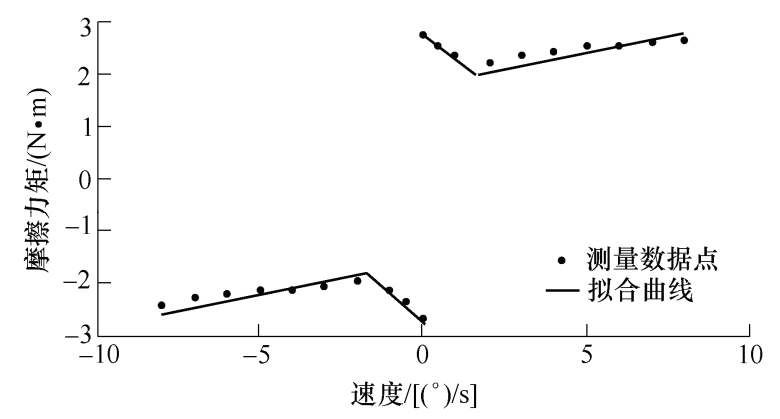

图 4 摩擦模型的曲线拟合

\section{2 动态摩擦特性参数辨识}

当系统处理临界运动或低速运动状态时, 系统 主要受库仑摩擦的影响, 有近似关系 $z \approx \theta$ 、 $\mathrm{d} z / \mathrm{d} t \approx \dot{\theta}$ 。

根据式(1), 可得近似系统动力学方程为

$$
J_{L} \ddot{\theta}+\left(\sigma_{1}+\sigma_{2}\right) \dot{\theta}+\sigma_{0} \theta=k_{T} i_{q}-m r \alpha_{J}
$$

式(18)近似为一个二阶阻尼系统, 其系数可通 过阶跃响应曲线拟合估计得到。当运动停止时, 有

$$
\sigma_{0} \approx \frac{T_{c} \operatorname{sgn}(\dot{\theta})}{\theta_{s}}
$$

式中, $\theta_{s}$ 为静摩擦区域的稳态角位移, 通过输入一 个介于 $T_{s} / k_{T}$ 和 $T_{c} / k_{T}$ 之间电流信号, 可得 $\theta_{s}$, 从 而根据式(19)确定 $\sigma_{0}$ 。

对于二阶系统，可得

$$
\sigma_{1}+\sigma_{2}=2 \xi \sqrt{J_{L} \sigma_{0}}
$$

式中, $\xi$ 为二阶系统的阻尼系统, 最佳取值为 $\xi=0.707$ 。根据稳态摩擦特性参数 $\sigma_{1}$, 可得 $\sigma_{2}$ 值。

综上, 可得摩擦力矩模型参数见表 1 。
表 1 摩擦模型参数

\begin{tabular}{lcc}
\hline \multicolumn{1}{c}{ 参数 } & 正转 & 反转 \\
\hline 库仓摩擦力矩 $T_{d} /(\mathrm{N} \cdot \mathrm{m})$ & -1.958 & 2.234 \\
最大静摩擦力矩 $T_{s} /(\mathrm{N} \cdot \mathrm{m})$ & -2.653 & 2.731 \\
斯特里贝克速度 $\dot{\theta}_{s} /\left[\left(^{\circ}\right) / \mathrm{s}\right]$ & -2.237 & 2.152 \\
黏滞摩擦因数 $\sigma_{2} /\left(\mathrm{N} \cdot \mathrm{m} /\left[\left(^{\circ}\right) / \mathrm{s}\right]\right)$ & 0.324 & 0.326 \\
刚度系数 $\sigma_{0} /\left[\mathrm{N} \cdot \mathrm{m} /\left(^{\circ}\right)\right]$ & 76.743 & 75.647 \\
阻尼系数 $\sigma_{1} /\left(\mathrm{N} \cdot \mathrm{m} /\left[\left(^{\circ}\right) / \mathrm{s}\right]\right)$ & 18.531 & 18.271 \\
\hline
\end{tabular}

\section{4 仿真分析和试验验证}

\section{1 试验平台及参数}

为了验证所提控制方法的有效性, 以某型机载 雷达伺服系统俯仰向跟踪控制为对象，进行传统 PID 控制与自适应摩擦补偿控制进行跟踪性能的 对比分析。硬件平台采用某型雷达伺服单元俯仰 轴向控制为试验研究对象。位置反馈器件采用旋 转变压器, 激磁电压 $7 \mathrm{~V} / 2 \mathrm{kHz}$ 、电气误差为 $3^{\prime}$ 。 逆变器采用 MSK4322 功率放大模块, 开关频率为 $10 \mathrm{kHz}$, 系统中通过 PWM 中断触发电流采样、位 置采样和占空比更新等。雷达伺服系统根据航电 模拟器给定的位置和速度指令信息进行相应的轨 迹扫描，同时实时将状态反馈信息发送给综合处 理单元进行目标搜索和跟踪计算。仿真和试验中 雷达伺服系统的参数如表 2 所示, 试验平台示意 图见图 5。

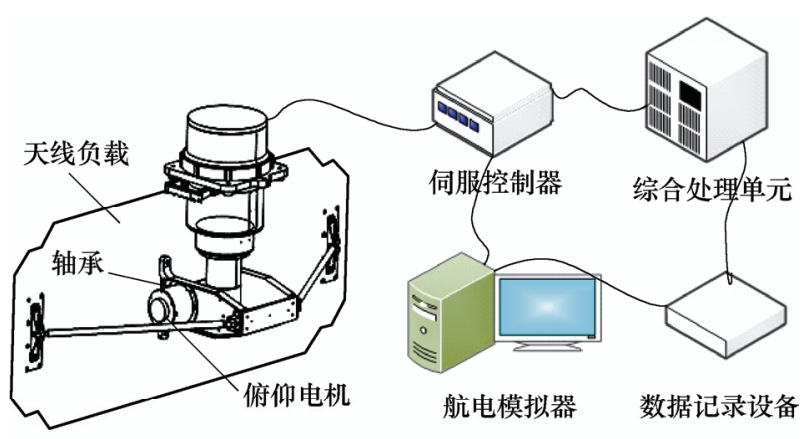

伺服机构

(a) 雷达试验平台示意图

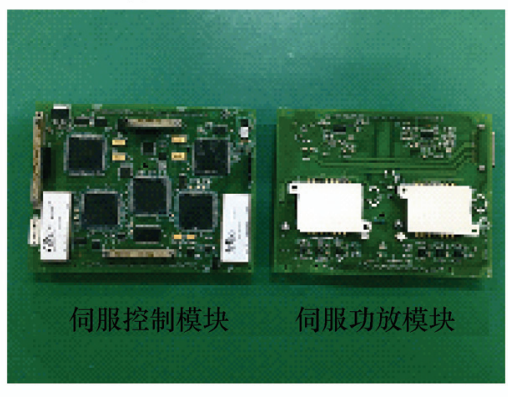

(b) 驱动模块实物图

图 5 雷达伺服系统试验平台 
表 2 系统参数

\begin{tabular}{cc}
\hline 参数名称 & 数值 \\
\hline 系统惯量 $/\left(\mathrm{kg} \cdot \mathrm{m}^{2}\right)$ & 2.317 \\
负载重量 $/ \mathrm{kg}$ & 14.5 \\
额定电压 $/ \mathrm{V}$ & 48 \\
额定转矩 $/ \mathrm{N} \cdot \mathrm{m})$ & 15 \\
额定转速 $/(\mathrm{r} / \mathrm{min})$ & 30 \\
定子电阻 $/ \Omega$ & 1.22 \\
直轴电感 $/ \mathrm{Mh}$ & 1.75 \\
交轴电感 $/ \mathrm{mH}$ & 1.75 \\
\hline
\end{tabular}

\section{2 仿真结果}

在 simulink 环境下搭建仿真模型, 设置系统采 样周期和计算步长均为 $0.1 \mathrm{~ms}$, 取自适应控制器增 益为 $r_{0}=30 、 r_{1}=15$, 摩擦模型参数取表 1 所示参 数。仿真结果如图 6 和图 7 所示。

图 6 为雷达工作于搜索状态时给定的正弦跟随 位置信号 $\theta(t)=10 \sin (0.5 \pi t)$, 分别采用 PI 控制和 自适应摩擦力矩控制时, 系统输出的角度跟随、跟 随误差、输出速度和 $q$ 轴电流波形。

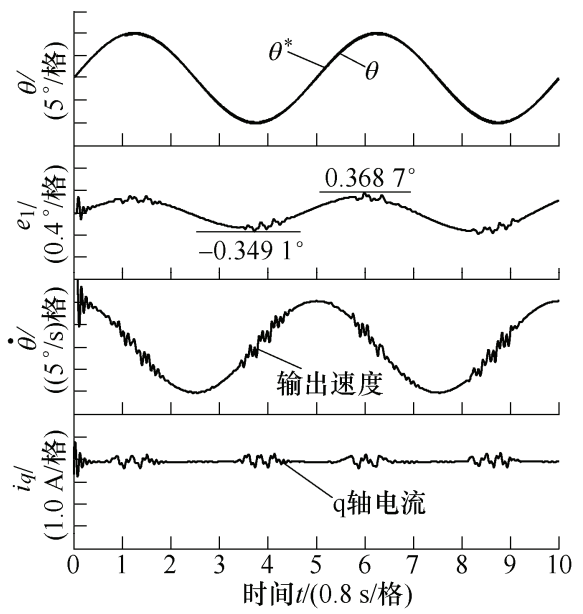

(a) PI控制

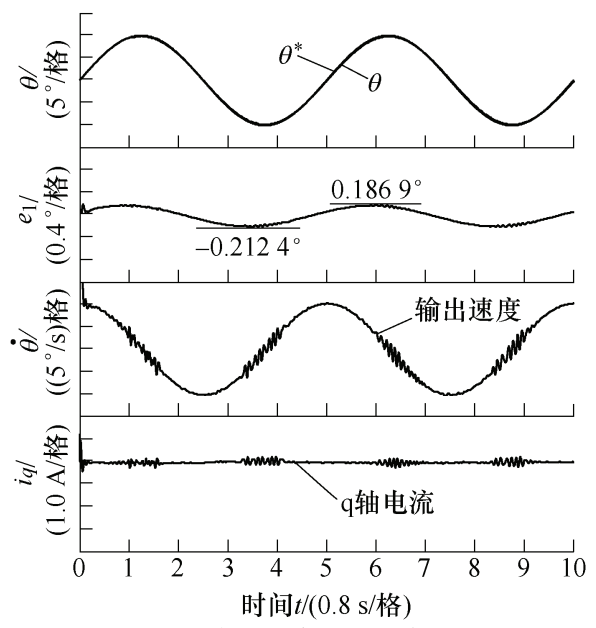

(b) 自适应摩擦补偿控制

图 6 正弦跟随仿真曲线

由图 6 表明, 伺服系统进行搜索工作时, 扫描
速度相对较大时, PI 控制和自适应摩擦补偿控制效 果相当; 当伺服机构进入机械换向时，此时扫描速 度接近零, 由于非线性摩擦力矩的影响, PI 控制的 跟踪误差变大, 转速和 $q$ 轴电流的脉动比较明显, 而采用自适应摩擦补偿控制的位置跟踪精度有明显 的提升，且转速和 $q$ 轴电流的波动也相对较小。

图 7 为模拟目标跟踪轨迹的跟踪位置信号 $\theta(t)=5 \sin (0.16 \pi t) \sin (0.09 \pi t)$ 分别采用 PI 控制和 自适应摩擦力矩控制时, 系统输出的角度跟随、跟 随误差、输出速度和 $q$ 轴电流波形。

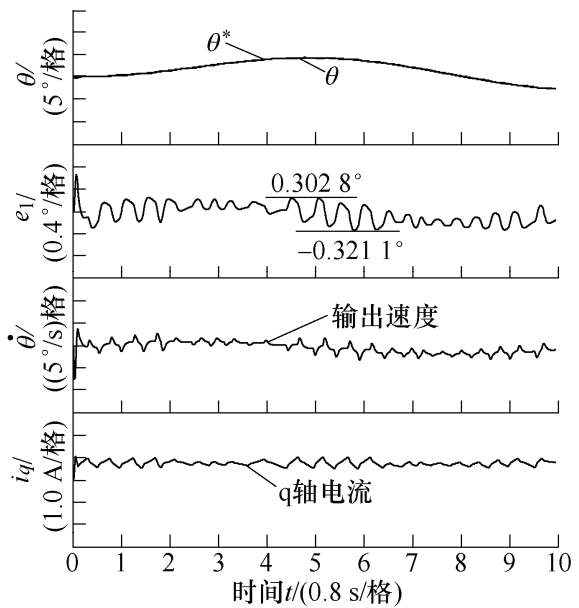

(a) PI控制

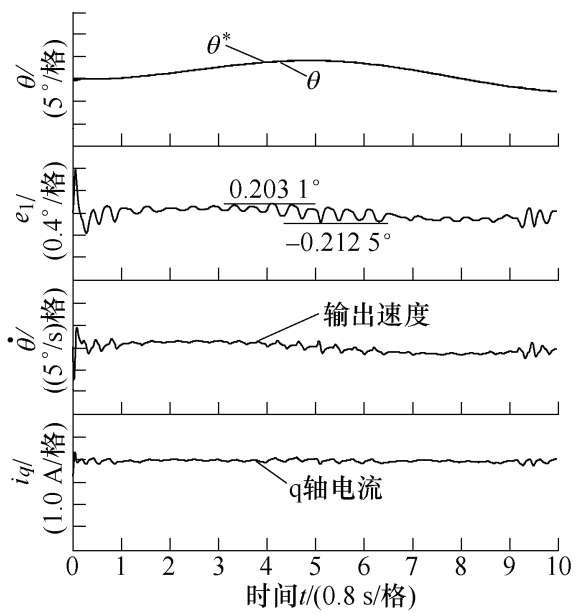

(b) 自适应摩擦补偿控制

图 7 低速跟踪仿真曲线

从图 7 可得, 在雷达伺服系统进行目标跟踪时, 跟踪速度低(小于 $5\left(^{\circ}\right) / \mathrm{s}$ ), 非线性摩擦力矩的影响比 较大, 相对于 PI 控制, 自适应摩擦补偿控制的跟踪 精度有明显改善。

\section{3 试验验证}

为验证以上仿真结果，本文在某型雷达伺服系 统中进行试验验证。试验中所用的控制参数与仿真 时相同。试验过程中通过航电仿真器给定俯仰正弦 搜索位置跟踪信号和模拟目标轨迹跟踪信号。试验 波形如图 8 和图 9 所示。 


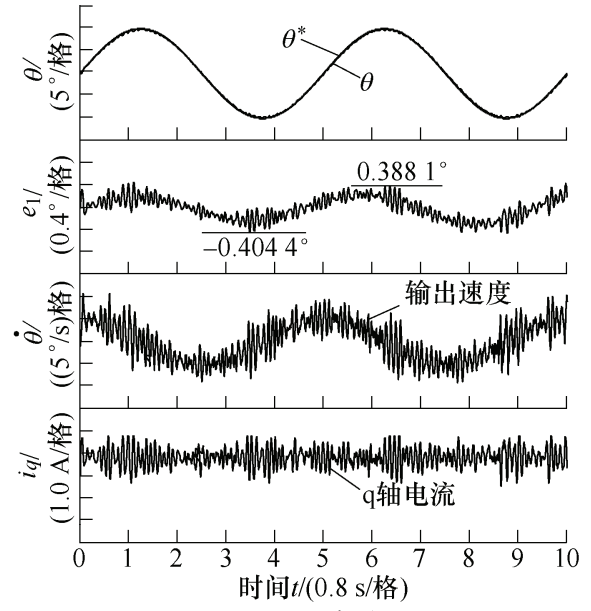

(a) PI控制

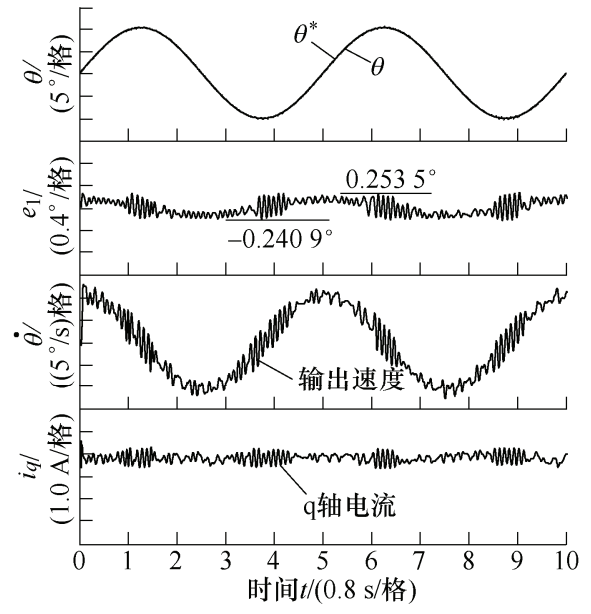

(b) 自适应摩擦补偿控制

图 8 正弦跟随试验曲线

由图 8 可得, 正弦搜索时, 扫描速度范围约 $5\left(^{\circ}\right) / \mathrm{s} \sim 10\left(^{\circ}\right) / \mathrm{s}$ 区域内, 转速相对平稳, 摩擦力矩的 处于线性区, 摩擦力矩对系统的影响相对较小, 自 适应摩擦力矩补偿控制和 PI 控制性能相近, 均能保 证系统良好的跟踪精度。当搜索状态进入机械换向 时, 进入扫描速度约小于 $5\left(^{\circ}\right) / \mathrm{s}$ 的低速扫描区域时, 此时跟踪精度误差变大, $q$ 轴电流有明显的脉动, 非线性摩擦力矩特性较明显。对比图 $8 \mathrm{a}$ 和 $8 \mathrm{~b}$ 可知, 相对于 PI 控制, 自适应摩擦力矩补偿控制的跟踪精 度有较大的提高。

图 9 为模拟目标跟踪曲线, 扫描速度较低 $\left(<5\left(^{\circ}\right) / \mathrm{s}\right)$, 整个跟踪过程中, 输出转速和 $q$ 轴电流 都有较大的波动, 摩擦力矩处于非线性区域, 对比 可知, 自适应摩擦力矩补偿控制在低速控制区域的 跟踪精度明显优于 PI 控制。

综上, 由仿真和试验数据对比表 3 可得, 自适 应摩擦补偿控制方法有效地补偿了低速跟踪和机械 换向时非线性摩擦力矩对系统的影响, 能够有效地 提高雷达伺服系统的跟踪精度, 且具有良好的抑制 摩擦扰动的能力。

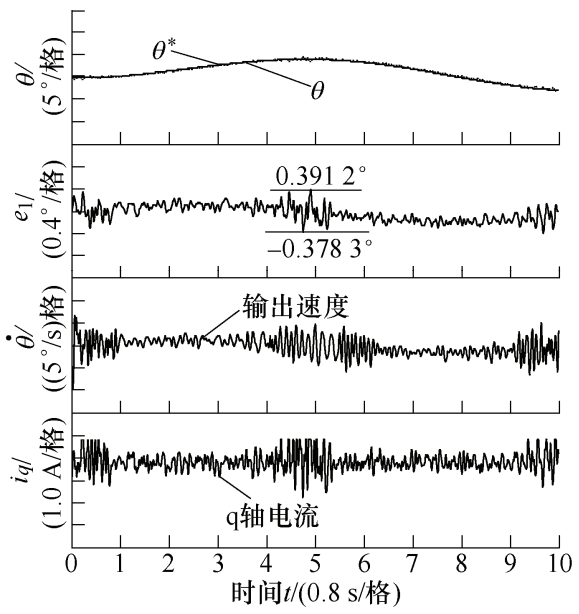

(a) PI控制

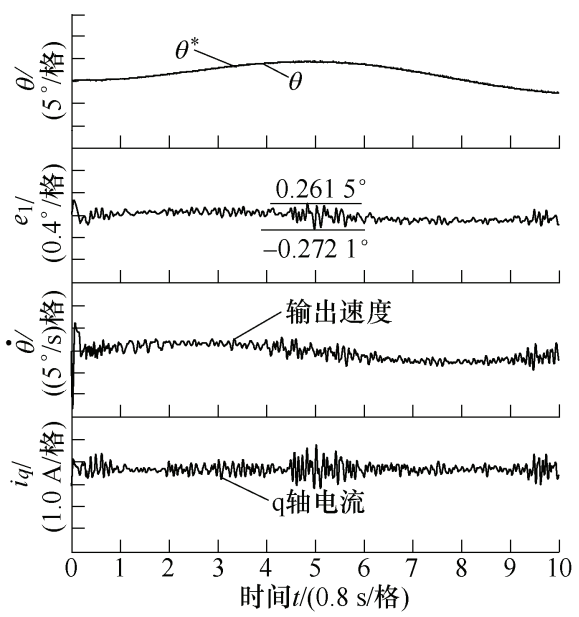

(b) 自适应摩擦补偿控制

图 9 低速跟踪试验曲线

表 3 仿真和试验数据对比

\begin{tabular}{llllll}
\hline \multirow{2}{*}{ 试验数据 } & \multicolumn{4}{c}{ 最大跟踪误差 $/\left({ }^{\circ}\right)$} \\
\cline { 3 - 6 } & & \multicolumn{2}{c}{ PI 控制 } & \multicolumn{3}{c}{ 摩擦补偿控制 } \\
\hline \multirow{2}{*}{ 仿真 } & 正弦跟随 & 0.3687 & -0.3491 & 0.1869 & -0.2124 \\
& 低速跟踪 & 0.3028 & -0.3211 & 0.2031 & -0.2125 \\
\hline \multirow{2}{*}{ 试验 } & 正弦跟随 & 0.3881 & -0.4044 & 0.2535 & -0.2049 \\
& 低速跟踪 & 0.3912 & -0.3783 & 0.2615 & -0.2721 \\
\hline
\end{tabular}

\section{5 结论}

雷达伺服系统在进行低速跟随和跟踪时, 摩擦 力矩非线性变化导致跟踪精度的下降, 严重时会导 致目标丢失。本文根据地面试验和试飞数据, 分析 了不同使用环境下，雷达伺服系统的摩擦力矩对伺 服跟踪精度的影响变化, 通过引入温度影响因子和 扰动影响因子, 改进了传统的 LuGre 动态摩擦模型, 设计了一种自适应摩擦力矩补偿控制策略, 并采用 李雅普诺夫稳定理论对所设计的自适应摩擦力矩补 偿控制策略进行稳定性证明, 得出控制参数的选择 范围。通过仿真和试验验证了该方法提高了雷达伺 
服系统在搜索和跟踪状态下的低速跟踪性能。结果 表明, 与传统 PI 控制策略相比, 自适应摩擦力矩补 偿控制策略的跟踪精度有明显的提高, 满足雷达伺 服系统恶劣工作环境下低速跟踪性能要求。

\section{参 考 文 献}

[1] CANUDAS D W C, OLSSON H, LISHINSKY P, et al. A new model for control of systems with friction[J]. IEEE Transactions on Automatic Control, 1995, 40(3): 419-425.

[2] ARMSTRONG H B, DUPONT P, CANUDAS D W C. A survey of models, analysis tools and compensation methods for the control of machines with friction[J]. Automatica, 1994, 30(7): 1083-1138.

[3] 刘栋, 梅雪松, 张东升. 基于多采样率控制的伺服系统 摩擦补偿研究[J]. 中国电机工程学报, 2011, 31(24): 111-117.

LIU Dong, MEI Xuesong, ZHANG Dongsheng. Friction compensator based on multi-rate control in servo drives system[J]. Proceedings of the CSEE, 2011, 31(24): 111-117.

[4] 袭著燕, 路长厚, 潘伟, 等. 基于预滑一动态摩擦力矩 估计模型的自适应前馈补偿方法 [J]. 机械工程学报, 2007, 43(10): 175-180.

XI Zhuyan, LU Changhou, PAN Wei, et al. Adaptive feed forward compensation method based on presliding-dynamic friction torque estimation model[J]. Journal of Mechanical Engineering, 2007, 43(10): 175-180.

[5] 吴跃飞, 马大为, 姚建勇, 等. 基于修正 LuGre 模型的 自适应鲁棒控制在机电伺服系统中的应用 $[\mathrm{J}]$. 机械工 程学报, 2014, 50(22): 207-212.

WU Yuefei, MA Dawei, YAO Jianyong, et al. Application of adaptive robust control in mechatronic servo system based on modified LuGre model[J]. Journal of Mechanical Engineering, 2014, 50(22): 207-212.

[6] 阎彦, 刘锐, 史婷娜, 等. 基于反推自适应控制的永磁 同步电机摩擦力矩补偿策略 [J]. 中国电机工程学报, 2013，33(33): 76-84.

YAN Yan, LIU Rui, SHI Tingna, et al. Friction compensation for permanent magnet synchronous motors based on adaptive back-steeping control[J]. Proceedings of CSEE, 2013, 33(33): 76-84.

[7] GUO Qing, YIN Jingmin, YU Tian, et al. saturated adaptive control of electrohydraulic actuator with parametric uncertainty and load disturbance[J]. IEEE Transactions on Industrial Electronics, 2017, 64(10): 7930-7941.

[8] 于爽, 付庄, 闰维新, 等. 基于干扰观测器的惯性平台 摩擦补偿方法[J]. 哈尔滨工业大学学报, 2008, 40(11): 1830-1833.
YU Shuang, FU Zhuang, YAN Weixin, et al. Method of friction compensation based on disturbance observer in inertial platform[J]. Journal of Harbin Institute of Technology, 2008, 40(11): 1830-1833.

[9] 王丽梅, 李兵. 基于摩擦观测器的直接驱动 $X Y$ 平台轮 廓控制器设计[J]. 电机与控制学报, 2013, 17(1): 31-36. WANG Limei, LI Bing. Contour controller design for direct $\mathrm{XY}$ table based on friction observer[J]. Electric Machines and Control, 2013, 17(1): 31-36.

[10] GUO Qing, ZHANG Yi, CELLER B G, et al. Backstepping control of electro-hydraulic system based on extended-state-observer with plant dynamics largely unknown[J]. IEEE Transactions on Industrial Electronics, 2016, 63(11): 6909-6920.

[11] GUO Q , SUN P , YIN J M. Parametric adaptive estimation and backstepping control of electro-hydraulic actuator with decayed memory filter.[J]. Isa Transactions, 2016, 62: 202-214.

[12] 过希文, 王群京, 李国丽, 等. 基于摩擦补偿的永磁球 形电机自适应模糊控制[J]. 中国电机工程学报, 2011, 31(15): 75-81.

GUO Xiwen, WANG Qunjing, LI Guoli, et al. Adaptive fuzzy control for permanent magnet spherical motor based on friction compensation[J]. Proceedings of the CSEE, 2011, 31(15): 75-81.

[13] 王永富, 王殿辉, 柴天佑. 基于状态估计的摩擦模糊建 模与鲁棒自适应控制 [J]. 自动化学报, 2011，37(2): 245-251.

WANG Yongfu, WANG Dianhui, CHAI Tianyou. State estimate-based friction fuzzy modeling and robust adaptive control[J]. Acta Automatica Sinica, 2011, 37(2): 245-251.

[14] 左月飞, 张捷, 刘闯, 等. 基于自抗扰控制的永磁同步 电机位置伺服系统一体化设计 $[\mathrm{J}]$. 电工技术学报, 2016, 31(11): 51-58.

ZUO Yuefei, ZHANG Jie, LIU Chuang, et al. Integrated design for permanent magnet synchronous motor servo systems based on active disturbance rejection control[J]. Transactions of China Electrotechnical Society, 2016, 31(11): 51-58.

[15] TAN K K, HUANG S N, LEE T H. Adaptive friction compensation with time-delay friction model[C]// Proceedings of the 4th World Congress on Intelligent Control and Automation, Shanghai, China, 2001: 969-973.

[16] SHIRIAEY A, ROBERTSSON A, JOHANSSON R. Friction compensation for nonlinear systems based on the LuGre model[C]// Proceedings of 16th IFAC-Symposium on Nonlinear Control Systems， 2005: 1223-1229.

[17] 谭文斌, 李醒飞, 赵新华, 等. 温度变化下的伺服系统 
非线性摩擦建模 $[J]$. 光学精密工程, 2014, 22(8): 2135-2141.

TAN Wenbin, LI Xingfei, ZHAO Xinhua, et al. Nonlinear friction modeling for servo systems in changed temperatures[J]. Optics and Precision Engineering, 2014, 22(8): 2135-2141.

[18] 魏琼, 焦宗夏, 李兴鲁. 考虑负载变化的气动摆动马达 LuGre 摩擦模型的研究 [J]. 液压与气动, 2014(2): 31-35. WEI Qiong, JIAO Zongxia, LI Xinglu. LuGre friction model of pneumatic motor with variable loader[J]. Chinese Hydraulics \& Pneumatics, 2014(2): 31-35.

[19] 姚建勇, 焦宗夏. 改进型 LuGre 模型的负载模拟器摩擦 补偿 [J]. 北京航空航天大学学报, 2010, 36(7): 812-820. YAO Jianyong, JIAO Zongxia. Friction compensation for hydraulic load simulator based on improved LuGre firction model[J]. Journal of Beijing University of Aeronautics and Astronautics, 2010, 36(7): 812-820.

[20] 丁千, 翟红梅. 机械系统摩擦动力学研究进展 [J]. 力学 进展, 2013，43(1): 112-131.
DING Qian, ZHAI Hongmei. The advance in researches of friction dynamics in mechanics system[J]. Advances in Mechanics, 2013, 43(1): 112-131.

[21] 向红标, 谭文斌, 李醒飞, 等. 基于 LuGre 模型的自 适应摩擦补偿[J]. 机械工程学报, 2012, 48(17): 70-74. XIANG Hongbiao, TAN Wenbin, LI Xingfei, et al. Adaptive Friction compensation based on lugre model[J]. Journal of Mechanical Engineering, 2012, 48(17): 70-74.

[22] SLOTINE J J E, LI W P. 应用非线性控制[M]. 北京: 机械工业出版社, 2009.

SLOTINE J J E, LI W P. Application of nonlinear control[M]. Beijing: China Machine Press, 2009.

作者简介: 姜仁华, 男, 1980 年出生, 博士研究生, 高级工程师。主要 研究方向为雷达交流伺服系统与智能控制。

E-mail: rhjiang@nuaa.edu.cn

刘闯, 男, 1973 年出生, 博士, 教授, 博士研究生导师。主要研究方向 为现代交流调速、开关磁阻电机、航空航天电源系统、新能源发电等技术。 E-mail: 1c@nuaa.edu.cn

宁银行, 男, 1982 年出生, 博士, 讲师。主要研究方向为现代交流调速、 新能源发电技术。

E-mail: nyh4711343@126.com 